\title{
Serological Evidence of SARS-CoV-2 Infection in Companion Animals in Pakistan: A Cross-sectional Survey
}

\author{
Aman Ullah ${ }^{1}$; Aitzaz Ahsan ${ }^{2}$; Siddra Tayyab Akhtar ${ }^{3}, \mathrm{PhD} ;$ Ambreen Chauhadry ${ }^{4}$; Muhammad Wasif Malik ${ }^{5}$, Mumtaz \\ Ali Khan ${ }^{5}$ Jamil A Ansari ${ }^{5}$; Aamer Ikram ${ }^{5}$ \\ ${ }^{1}$ Khan Bahadar Choudhry Mushtaq Ahmed College of Veterinary and Animal Sciences, University of Veterinary and Animal Sciences, Narowal, \\ Pakistan \\ ${ }^{2}$ Animal Health Program, Animal Sciences Institute, National Agricultural Research Centre, Islamabad, Pakistan \\ ${ }^{3}$ Institute of Microbiology and Molecular Genetics, The University of Punjab, Lahore, Pakistan \\ ${ }^{4}$ Field Epidemiology and Laboratory Training Program, National Institute of Health, Islamabad, Pakistan \\ ${ }^{5}$ National Institute of Health, Islamabad, Pakistan
}

\section{Corresponding Author:}

Aman Ullah

Khan Bahadar Choudhry Mushtaq Ahmed College of Veterinary and Animal Sciences

University of Veterinary and Animal Sciences

$5 \mathrm{Km}$, Shakargarh Road

Narowal

Pakistan

Phone: 923115000166

Email: aman.ullah@uvas.edu.pk

\section{Abstract}

Background: Coronaviruses infect both humans and animals. One of these viruses, SARS-CoV-2, associated with the COVID-19 pandemic, is believed to be zoonotic in nature with its origins from live animal markets in Wuhan, China. The virus has been reported to transmit from humans to other animals and within animal species both naturally and experimentally in many countries. Being a novel virus, studies are needed to understand how it affects animals, possible spread to humans, and other potential roles in the epidemiology of COVID-19 following a one health approach.

Objective: Therefore, we conducted a cross-sectional sero-survey among companion animals (pet dogs and cats) in twin cities of Islamabad and Rawalpindi, Pakistan with the aim to determine the seroprevalence of COVID-19 among these animals.

Methods: The study was conducted from November 2020 to April 2021, coinciding with the end of the second wave and the peak of the third wave of COVID-19 in Pakistan. We collected serum samples from 83 animals (68 dogs and 15 cats) from 10 small animal clinics and surgeries in twin cities. The data were collected on the species, age, sex, clinical history, travel history, and confirmation of COVID-19 among the owners or their families, presented with various clinical histories. The samples were tested by ID Screen SARS-CoV-2 double antigen multispecies enzyme-linked immunosorbent assay developed by ID.Vet, France.

Results: A combined seroprevalence of COVID-19 among these companion animals of $7.23 \%$ (6/83; 95\% CI 2.7\%-15.07\%) was found. Further, the seroprevalence of COVID-19 among dogs and cats were $7.35 \%$ (5/68; 95\% CI 2.43\%-16.33\%) and 6.67\% $(1 / 15 ; 95 \%$ CI $0.17 \%-31.95 \%)$, respectively. Both species were found to be similarly susceptible to COVID-19 (odds ratio 1.11 , $95 \%$ CI $0.12-10.27 ; P=.92)$. Animals in families with a history of COVID-19 among owners were more likely to be seropositive for SARS-CoV-2 (odds ratio $11.8,95 \%$ CI 1.93-71.89; $P=.002$ ).

Conclusions: The results suggest a possible transmission of SARS-CoV-2 from pet owners to their companion animals. However, further studies may be needed to evaluate this hypothesis and the role of pets as potential reservoirs for SARS-CoV-2 infection for humans and other animals.

(iproc 2022;8(1):e36534) doi: 10.2196/36534

\section{KEYWORDS}

COVID-19; dogs; cats; Pakistan; serology 


\section{Multimedia Appendix 1}

Serological evidence of SARS-CoV-2 infection in companion animals in Pakistan. [PPTX File, 294 KB-Multimedia Appendix 1]

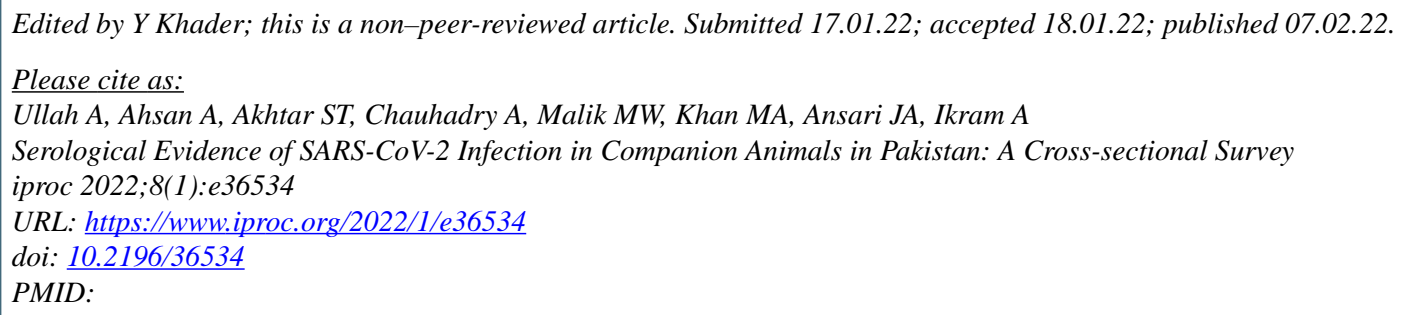

(C)Aman Ullah, Aitzaz Ahsan, Siddra Tayyab Akhtar, Ambreen Chauhadry, Muhammad Wasif Malik, Mumtaz Ali Khan, Jamil A Ansari, Aamer Ikram. Originally published in Iproceedings (https://www.iproc.org), 07.02.2022. This is an open-access article distributed under the terms of the Creative Commons Attribution License (https://creativecommons.org/licenses/by/4.0/), which permits unrestricted use, distribution, and reproduction in any medium, provided the original work, first published in Iproceedings, is properly cited. The complete bibliographic information, a link to the original publication on https://www.iproc.org/, as well as this copyright and license information must be included. 